\title{
CBCT EVALUATION OF CEPHALOMETRIC VARIABLES AMONG SAMPLES OF EGYPTIAN NON-OPERATED CLEFT LIP \\ AND PALATE PATIENTS
}

\author{
Ayman R. Khalifa', Engy Ali Abdelhaleem Salam ${ }^{2}$
}

Keywords: cleft lip and palate, CBCT, cephalometric analysis.

\section{ABSTRACT}

Objectives: to evaluate the cephalometric and craniofacial measurements in unilateral and bilateral cleft lip and palate (CLP) patients and to investigate the effect of these measurements in growing and nongrowing cleft patients using cone beam computed tomography (CBCT) 3D software, and to compare them to healthy individual.

Setting and Sample population: Retrospective study was performed in Department of pedodontics and Orthodontics, October 6 University. The study sample comprised unilateral and bilateral 36 growing $C \mathcal{L} \mathscr{P}$ (aged 6-17years), and 24 non-growing CLP (aged 1840 years). The control group included 60 Non-CLP subjects.

Methods: Cephalometric measurements were obtained using CBCT software, all measurements were assessed 6y Mann-test to clarify differences between study samples.

Results: There was a significant difference between (Growing Bilateral) and (Control Growing) for (MP$S \mathcal{N}, \mathcal{B}, \mathcal{A}, \mathcal{A N} \mathcal{N}, S \mathcal{N} B$ Q $\mathcal{A} \mathcal{A N S}-\mathcal{M} E)$ and There was a significant difference between (Growing Unilateral) and (Control Growing) for (MPP-SN, B, SNA, SNB,

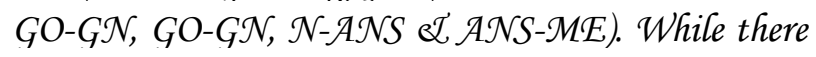
was a significant difference between (Non-Growing Bilateral) and (Control Non-Growing) for (MP-SN, $\mathscr{B}, \mathcal{A}, S \mathcal{N} \mathcal{A}, S \mathcal{N} B, G O-G \mathcal{N}, \mathcal{Q} \mathcal{L} \mathcal{A N S}-\mathcal{M E}$ ) , and there was a significant difference between (Non-Growing Unilateral) and (Control Non-Growing) for (MPP-SN,

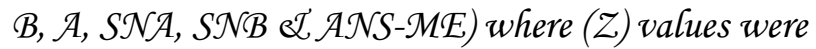
significant at (0.05).

Conclusion: Our study shows that there was significant increase in mandibular length in 6oth growing and non-growing $B C L P$ than of $U C \mathcal{L}$, in growing UCLP there was reduced upper face height and in growing $B C L \mathcal{P}$ there was increased lower face height

\section{Introduction}

There are many Craniofacial deformities and defects which may be challenged and compromised many distinct oral health-related problems ${ }^{[1]}$. Among them is the cleft lip or/and palate (CLP) which consider the most common congenital anomaly in the head and neck area ${ }^{[2,3]}$. The incident of CLP has been documented as 0.18 per 1000 live birth around the world ${ }^{[3,4]}$. Abulezz et al, 2018 reported that it is suspected to have more than 3000 new cleft cases per year in Egypt and the incident of cleft is $1 / 750$ live birth ${ }^{[5]}$.

The management of cleft patient needs interdisciplinary approach including plastic surgery, speech pathology, orthodontics, audiology, pediatrics, anesthesiology, dentistry, otolaryngology, psychology and genetics [6, ${ }^{7]}$.The impacts on growth, function, appearance and psychological outcome must be considered in assessing the treatment outcomes of cleft patients ${ }^{[8]}$.

There are well known variations among cleft patients in growth patterns and measurements in the vertical, antero-posterior and transverse dimensions leading to dental malocclusion. The development and shape of the maxillary arch may be affected as a result of the variation of the initial cleft deformity ${ }^{[9]}$.

Many studies have shown that patients with more sever cleft in the palate/ alveolar bone or greater palate tissue deficiency indicates less beneficial development of the maxillary structure and Poorer relationships

\footnotetext{
${ }^{1}$ Department of Pedodontics and Orthodontics, October 6 University, Faculty of Dentistry, Giza, Egypt

${ }^{2}$ Department of oral and maxillofacial radiology, Faculty of Dentistry, Fayoum University.
} 
between dental arches ${ }^{[10-15]}$. On the other hand, other studies do not support this theory ${ }^{[16-19]}$.

2D cephalometric radiographic tracing remained the main diagnostic method of choice in orthodontics since many decades ${ }^{[20-23]}$. However, there is a usual demand to additional radiograph in other direction to evaluate malocclusion and facial asymmetry ${ }^{[22]}$.

The evolution of Cone beam computed tomography (CBCT) many years ago, overcomes the high cost and radiation dosage of medical CT and it allows three-dimensional (3D) visualization of oral and maxillofacial complex and metal-induced artifacts, and higher spatial resolution ${ }^{\text {[24-27] }}$

CBCT has dramatically improved imaging within the dental field, it provides accurate visualization of orthodontic problems, aiding in the planning and evaluation of orthodontic treatment. In the last 20 years, the use of (CBCT) has created new possibilities for more effective and accurate sagittal assessment. CBCT offers accurate and distortion-free image of craniofacial structure without any issues with magnification or superimposition, $^{[22,28]}$ so it can be used for cephalometric analyses and planning of orthognathic surgery, orofacial clefts, craniofacial syndromes, soft and hard tissue asymmetries, and nasopharyngeal airway abnormalities [29-32]. Where, Lateral cephalograms can be obtained easily from CBCT scans as reconstructed lateral cephalometric radiographs (RLCs). The accuracy of linear and angular measurements evaluated with lateral cephalograms and RLCs showed no significant difference ${ }^{[33]}$.

Previous studies indicate that the overall accuracy and reproducibility of the position of the different CBCT landmarks widely used for orthodontic diagnosis is high ${ }^{[34-37]}$. The American Association of Orthodontists (AAO) has revised its clinical practice guidelines for 3D cone beam computed tomography (CBCT) as part of the patient's medical history and diagnostic guide $^{[36]}$
The aim of this study is to evaluate the cephalometric and craniofacial measurements in unilateral and bilateral CLP Egyptian patients and to investigate the effect of these measurements in growing and non-growing cleft patients using CBCT 3D software, and to compare them to healthy individual.

\section{Material and methods}

This study sample included 60 CLP patients (26 males - 34 females), while the control group included 60 Non-CLP subjects, patients, who visited the Department of pedodontics and Orthodontics, October 6 University. The exclusion criteria were history of orthognathic surgical repair, history of any type of syndrome or craniofacial anomalies. All individuals with CLP and control groups had class I malocclusion with no previous orthodontic intervention. The sample was classified into four groups, according to cleft type (unilateral or bilateral cleft) and subject age (growing or non-growing).

For growing cleft group, 36 subjects (with age range between 6-17 years old) was subdivided to unilateral growing (UGCLP) and bilateral growing (BGCLP) groups (15 and 21 subjects respectively). And for non-growing cleft group, 24 subjects (with age 18-40 years old) also were subdivided to unilateral non-growing (UNGCLP) and bilateral non-growing (BNGCLP) groups (10 and 14 subject respectively). The control group was subdivided to growing and non-growing control group (30 and 30 subjects).

This study was approved by the Ethics Committee at Faculty of Dentistry October 6 University (RECO6U/9-2019).

CBCT examination was performed for patients with and without clefts scheduled to undergo one of several procedures or evaluations: alveolar bone grafting, orthodontic treatment, or removal of impacted teeth; evaluation of skeletal Class III malocclusion; or evaluation of anterior open bite and lateral crossbite.

All CBCT scans were performed with patients in maximum dental intercuspation with the IICAT machine (Imaging Systems, CITY) with 
a slice thickness of $0.3 \mathrm{~mm}$ at $1024 \times 1024$ resolution. Scanning time was 20 seconds. Head position was standardized by adjusting the chin rest and bite block to the patient's height and then aligning on the Frankfurt plane.

\section{CBCT Analysis:}

Cone beam computed tomography images were converted to Dicom format and processed into volumetric images using Anatomage image processing software version 5.01g. Multiplanar sagittal, coronal and axial projections were generated. All images were oriented in the sagittal, axial, and coronal planes before taking measurements by adjusting the midsagittal plane to pass through the skeletal midline of the face (Nasion Subnasale), the axial plane was adjusted with the Frankfort horizontal plane (Porion -
Orbitale), and the coronal plane was adjusted to pass through the level of the furcation point of the maxillary right first molar. A Cephalometric landmarks and parameters were analyzed by the software according $\mathrm{ABO}$ guidelines Tables $(1,2)$, Fig.1.

Statistical analysis of survey methods:

Data were unloaded by known Statistical Package for Social Sciences Statistical Program was the statistical analysis using the computer through a statistical package software SPSS V. 25 and through it, we used the following tests:

1. Descriptive statistics of the data through the tabulating data in the form of (frequencies, proportion, means and standard deviation).

2. Mann-Whitney Test to clarify the differences between the study sample.

Table I Definitions of landmarks and cephalometric parameters used in the study.

\begin{tabular}{|l|l|}
\hline Nasion (N): & Point where frontonasal and internasal sutures meet in the midline \\
\hline $\begin{array}{l}\text { Anterior nasal } \\
\text { spine (ANS): }\end{array}$ & Most anterior point of maxilla; used for vertical measurement. \\
\hline $\begin{array}{l}\text { Point A } \\
\text { (subspinale): }\end{array}$ & $\begin{array}{l}\text { The deepest point in the curvature between ANS and inferior most point in the } \\
\text { maxillary alveolar process. }\end{array}$ \\
\hline $\begin{array}{l}\text { Point B } \\
\text { (supramentale): }\end{array}$ & $\begin{array}{l}\text { The deepest point in the curvature between pogonion and superior most point in } \\
\text { the mandibular alveolar process }\end{array}$ \\
\hline Gnathion (Gn) & $\begin{array}{l}\text { The most anterior and inferior point on the lateral shadow of chin. It is } \\
\text { approximately in the midpoint between pogonion and menton. }\end{array}$ \\
\hline Menton (Me) & The inferior most point in the contour of the chin. \\
\hline Basion (Ba) & $\begin{array}{l}\text { The most posterior and inferior point in the sagittal plane on the anterior rim of } \\
\text { foramen magnum. }\end{array}$ \\
\hline Gonion (Go): & The most posterior and inferior point at the angle of mandible. \\
\hline Sella (S): & Centre of the pituitary fossa or sella turcica. Bilateral points \\
\hline $\begin{array}{l}\text { SN Plane } \\
\text { S-N }\end{array}$ & $\begin{array}{l}\text { This plan is represents the anterior cranial base and is formed by connecting } \\
\text { Sella to Nasion }\end{array}$ \\
\hline $\begin{array}{l}\text { Mandibular } \\
\text { Plane (MP) } \\
\text { Go-Gn }\end{array}$ & $\begin{array}{l}\text { This plane is formed by connecting the point Gonion to Gnathion at the inferior } \\
\text { border of the mandible }\end{array}$ \\
\hline $\begin{array}{l}\text { A point } \\
\text { (subspinale) }\end{array}$ & Most concave point of anterior maxilla \\
\hline $\begin{array}{l}\text { B point } \\
\text { supramentale }\end{array}$ & Most concave point on mandibular symphysis \\
\hline $\begin{array}{l}\text { Basion } \\
\text { Ba) }\end{array}$ & Most anterior point on foramen magnum \\
\hline $\begin{array}{l}\text { Gnathion }(\mathrm{Gn}) \\
\text { pogonion and menton }\end{array}$ & $\begin{array}{l}\text { Point located perpendicular on mandibular symphysis midway between } \\
\text { pang }\end{array}$ \\
\hline
\end{tabular}




\begin{tabular}{|l|l|}
\hline \multicolumn{1}{|c|}{$\begin{array}{l}\text { Gonion } \\
\mathrm{GO})\end{array}$} & $\begin{array}{l}\text { Most posterior inferior point on angle of mandible. Can also be constructed by } \\
\text { bisecting the angle formed by intersection of mandibular plane and ramus of } \\
\text { mandible }\end{array}$ \\
\hline $\begin{array}{l}\text { Anterior Nasal } \\
\text { Spine }(\text { ANS })\end{array}$ & Anterior point on maxillary bone \\
\hline Menton $(\mathrm{Me})$ & Lowest point on mandibular symphysis \\
\hline SNA $\left(^{\circ}\right)$ & Sella-Nasion to A Point Angle \\
\hline SNB $\left(^{\circ}\right)$ & Sella-Nasion to B Point Angle \\
\hline N vertical & Line extended from Nasion perpendicular to Frankfurt horizontal plane FH \\
\hline N-ANS & Linear measurement from Nasion to ANS \\
\hline $\begin{array}{l}\text { A point } \\
\text { measurement }\end{array}$ & Linear measurement from A point to N vertical \\
\hline $\begin{array}{l}\text { B point } \\
\text { measurement }\end{array}$ & Linear measurement from B point to N vertical \\
\hline
\end{tabular}

Table 2 Definitions of variable cephalometric parameters used in the study

\begin{tabular}{|c|c|c|}
\hline $\begin{array}{l}\text { Linear and Angular } \\
\text { Landmark }\end{array}$ & Definition & Measures \\
\hline MP-SN(angular ) & $\begin{array}{l}\text { Mandibular plane and anterior } \\
\text { cranial base }\end{array}$ & $\begin{array}{l}\text { Denote the growth pattern mean } 32 \\
\text { degree }\end{array}$ \\
\hline ANB (angular) & $\begin{array}{l}\text { The angle between point } A, N \\
\text { (nasion) and } B\end{array}$ & $\begin{array}{l}\text { Correlate position of maxilla to } \\
\text { mandible }\end{array}$ \\
\hline SNA (angular) & $\begin{array}{l}\text { The angle between point } A \text { and } \\
\text { anterior cranial base (angular) }\end{array}$ & $\begin{array}{l}\text { Correlate position of maxilla to } \\
\text { cranial base }\end{array}$ \\
\hline SNB (angular) & $\begin{array}{l}\text { The angle between point } B \text { and } \\
\text { anterior cranial base }\end{array}$ & $\begin{array}{l}\text { Correlate position of mandible to } \\
\text { cranial base }\end{array}$ \\
\hline GO-GN (linear) & Gonion to gnathion & Mandibular length \\
\hline Bs-S-N (angular) & Basion -Sella-Nasion & Denote cranial base divergence \\
\hline N-ANS & $\begin{array}{l}\text { Nasion to anterior nasal } \\
\text { spine(linear) }\end{array}$ & Upper Anterior facial height \\
\hline ANS-Me (linear) & Anterior nasal spine to Menton & Lower Anterior facial height \\
\hline
\end{tabular}
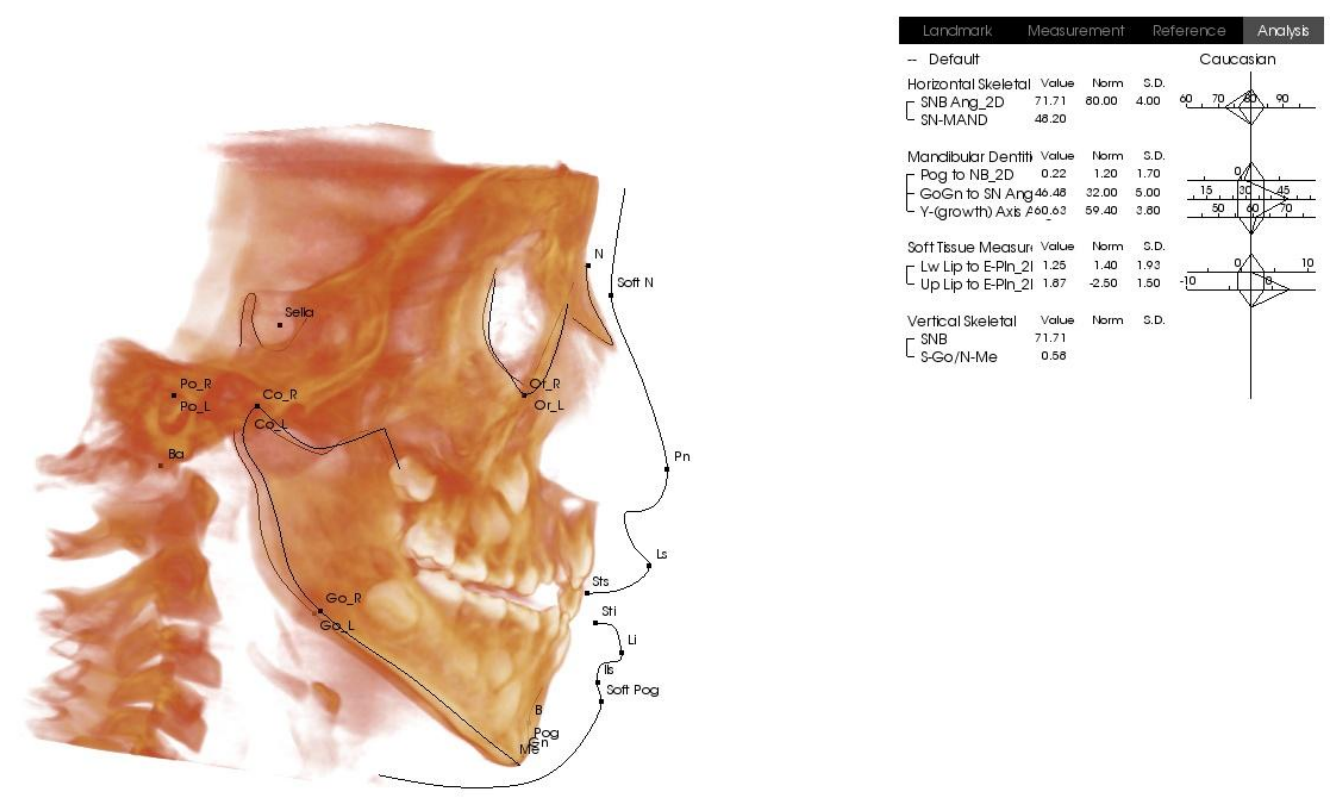

Fig (1): lateral 3D cephalometric analysis 


\section{Results}

The sample used is called convenience sampling including: Cases groups were Growing Bilateral 21(17.5\%) (male) (8) (6.7\%) and (female) (13) (10.8\%). Growing Unilateral was $15(12.5 \%)$, (male) (8) $(6.7 \%)$ and (female) (7) $(5.8 \%)$. Non-Growing Bilateral was $10(8.3 \%)$ (male) (4) (3.3\%) and (female) (6)
(5.0\%). Non-Growing Unilateral was $14(11.7 \%)$ (male) (5) (4.2\%) and (female) (9) $(7.5 \%)$. Control groups were Control Growing was 30(25.0\%) (male) (12) (10.0\%) and (female) (18) $(15.0 \%)$. Control Non-Growing was 30(25.0\%) (male) (12) (10.0\%) and (female) (18) (15.0\%).

Table (3): Comparison between Growing Bilateral Group and control Growing group \& Comparison between Growing Unilateral Group and control Growing group

\begin{tabular}{|c|c|c|c|c|c|c|c|c|c|c|c|c|}
\hline \multirow{2}{*}{$\begin{array}{c}\begin{array}{c}\text { Variabl } \\
\text { e }\end{array} \\
\text { MP-SN }\end{array}$} & \multicolumn{2}{|c|}{$\begin{array}{c}\text { Growing } \\
\text { Bilateral } \\
(\mathrm{n}=21) \\
\text { Mean } \pm \text { SD }\end{array}$} & \multicolumn{2}{|c|}{$\begin{array}{c}\text { Control } \\
\text { Growing } \\
(\mathbf{n}=30) \\
\text { Mean } \pm \text { SD }\end{array}$} & \multirow{2}{*}{$\begin{array}{c}\mathbf{Z} \\
4.251\end{array}$} & \multirow{2}{*}{$\begin{array}{c}\begin{array}{c}\text { P- } \\
\text { value }\end{array} \\
0.001 \\
\text { H.S }\end{array}$} & \multicolumn{2}{|c|}{$\begin{array}{c}\text { Growing } \\
\text { Unilateral } \\
(\mathbf{n}=15) \\
\text { Mean } \pm \\
\text { SD }\end{array}$} & \multicolumn{2}{|c|}{$\begin{array}{c}\text { Control } \\
\text { Growing } \\
(\mathbf{n}=30) \\
\text { Mean } \pm \\
\text { SD }\end{array}$} & \multirow{2}{*}{$\begin{array}{c}\mathbf{Z} \\
5.013\end{array}$} & \multirow{2}{*}{$\begin{array}{c}\begin{array}{c}\text { P- } \\
\text { value }\end{array} \\
0.001 \\
\text { H.S }\end{array}$} \\
\hline & $\begin{array}{c}41.1 \\
4\end{array}$ & 8.63 & 32.81 & $\begin{array}{c}4.2 \\
8\end{array}$ & & & $\begin{array}{c}47.2 \\
4\end{array}$ & $\begin{array}{c}8.2 \\
8\end{array}$ & $\begin{array}{c}32.8 \\
1\end{array}$ & $\begin{array}{c}4.2 \\
8\end{array}$ & & \\
\hline B & -2.60 & $\begin{array}{c}10.5 \\
4\end{array}$ & -1.47 & $\begin{array}{c}3.5 \\
3\end{array}$ & 2.011 & $0.04 \mathrm{~S}$ & -5.80 & $\begin{array}{c}7.5 \\
5\end{array}$ & -1.47 & $\begin{array}{c}3.5 \\
3\end{array}$ & 3.085 & $\begin{array}{c}0.002 \\
\text { H.S }\end{array}$ \\
\hline A & 5.05 & 7.62 & 2.27 & $\begin{array}{c}1.9 \\
8\end{array}$ & 2.016 & $0.04 \mathrm{~S}$ & 0.43 & $\begin{array}{c}5.6 \\
8\end{array}$ & 2.27 & $\begin{array}{c}1.9 \\
8\end{array}$ & 0.822 & $\begin{array}{c}0.4 \\
\text { N.S }\end{array}$ \\
\hline ANB & 8.12 & 6.03 & 2.49 & $\begin{array}{c}2.6 \\
7\end{array}$ & 3.850 & $\begin{array}{c}0.001 \\
\text { H.S }\end{array}$ & 3.17 & $\begin{array}{c}4.0 \\
2\end{array}$ & 2.49 & $\begin{array}{c}2.6 \\
7\end{array}$ & 0.434 & $\begin{array}{c}0.7 \\
\text { N.S }\end{array}$ \\
\hline SNA & $\begin{array}{c}82.7 \\
2\end{array}$ & 7.14 & 80.57 & $\begin{array}{c}2.8 \\
8\end{array}$ & 0.919 & $\begin{array}{c}0.4 \\
\text { N.S }\end{array}$ & $\begin{array}{c}76.4 \\
3\end{array}$ & $\begin{array}{c}5.2 \\
2\end{array}$ & $\begin{array}{c}80.5 \\
7\end{array}$ & $\begin{array}{c}2.8 \\
8\end{array}$ & 2.723 & $\begin{array}{c}0.006 \\
\text { H.S }\end{array}$ \\
\hline SNB & $\begin{array}{c}74.6 \\
0\end{array}$ & 5.39 & 78.08 & $\begin{array}{c}3.2 \\
2\end{array}$ & 3.084 & $\begin{array}{c}0.002 \\
\text { H.S }\end{array}$ & $\begin{array}{c}73.2 \\
5\end{array}$ & $\begin{array}{c}4.7 \\
6\end{array}$ & $\begin{array}{c}78.0 \\
8\end{array}$ & $\begin{array}{c}3.2 \\
2\end{array}$ & 3.229 & $\begin{array}{c}0.001 \\
\text { H.S }\end{array}$ \\
\hline GO-GN & $\begin{array}{c}70.4 \\
7\end{array}$ & 8.48 & 68.54 & $\begin{array}{c}4.3 \\
9\end{array}$ & 0.517 & $\begin{array}{r}0.6 \\
\text { N.S }\end{array}$ & $\begin{array}{c}75.4 \\
3\end{array}$ & $\begin{array}{c}7.6 \\
6\end{array}$ & $\begin{array}{c}68.5 \\
4\end{array}$ & $\begin{array}{c}4.3 \\
9\end{array}$ & 3.374 & $\begin{array}{c}0.001 \\
\text { H.S }\end{array}$ \\
\hline BS-S-N & $\begin{array}{c}128 . \\
93\end{array}$ & 7.16 & 132.13 & $\begin{array}{c}4.8 \\
3\end{array}$ & 1.436 & $\begin{array}{r}0.2 \\
\text { N.S }\end{array}$ & $\begin{array}{c}128 . \\
85\end{array}$ & $\begin{array}{c}8.7 \\
3\end{array}$ & $\begin{array}{c}132 . \\
13\end{array}$ & $\begin{array}{c}4.8 \\
3\end{array}$ & 1.132 & $\begin{array}{c}0.3 \\
\text { N.S }\end{array}$ \\
\hline N-ANS & $\begin{array}{c}52.4 \\
6\end{array}$ & 7.46 & 48.82 & $\begin{array}{c}2.4 \\
439 \\
1\end{array}$ & 1.935 & $\begin{array}{c}0.053 \\
\text { N.S }\end{array}$ & $\begin{array}{c}55.8 \\
1\end{array}$ & $\begin{array}{c}7.5 \\
4\end{array}$ & $\begin{array}{c}48.8 \\
2\end{array}$ & $\begin{array}{c}2.4 \\
4\end{array}$ & 3.688 & $\begin{array}{c}0.001 \\
\text { H.S }\end{array}$ \\
\hline $\begin{array}{l}\text { ANS- } \\
\text { ME }\end{array}$ & $\begin{array}{c}69.4 \\
5\end{array}$ & $\begin{array}{c}12.9 \\
7\end{array}$ & 60.93 & $\begin{array}{c}5.2 \\
0\end{array}$ & 2.681 & $\begin{array}{c}0.007 \\
\text { H.S }\end{array}$ & $\begin{array}{c}74.5 \\
7\end{array}$ & $\begin{array}{l}10 . \\
70\end{array}$ & $\begin{array}{c}60.9 \\
3\end{array}$ & $\begin{array}{c}5.2 \\
0\end{array}$ & 4.361 & $\begin{array}{c}0.001 \\
\text { H.S }\end{array}$ \\
\hline
\end{tabular}

From the table (3) showed the following:

There was a significant difference at $\mathrm{p}$-value (0.05) between (Growing Bilateral) and (Control Growing) for (MP-SN, B, A, ANB, $\mathrm{SNB} \&$ ANS-ME) where (Z) values were significant at (0.05).

There was non-significant difference at p-value (0.05) between (Growing Bilateral) and (Control Growing) for (SNA, GO-GN, BS-S-N \& N-ANS) where (Z) values were nonsignificant at (0.05).
There was a significant difference at $\mathrm{p}$-value (0.05) between (Growing Unilateral) and (Control Growing) for (MP-SN, B, SNA, SNB, GO-GN, GO-GN, N-ANS \& ANS-ME) where $(Z)$ values were significant at (0.05).

There was non-significant difference at p-value (0.05) between (Growing Unilateral) and (Control Growing) for (A, ANB \& BS-S-N) where $(\mathrm{Z})$ values were non-significant at (0.05). 
Table (4): Comparison Between Non-Growing Bilateral Group and control Non-Growing group \& Comparison between Non-Growing Unilateral Group and control Non-Growing group

\begin{tabular}{|c|c|c|c|c|c|c|c|c|c|c|c|c|}
\hline \multirow{2}{*}{$\begin{array}{c}\begin{array}{c}\text { Variabl } \\
\text { e }\end{array} \\
\text { MP-SN }\end{array}$} & \multicolumn{2}{|c|}{$\begin{array}{c}\text { Non- } \\
\text { Growing } \\
\text { Bilateral } \\
(n=10) \\
\text { Mean } \pm \text { SD }\end{array}$} & \multicolumn{2}{|c|}{$\begin{array}{c}\text { Control } \\
\text { Non- } \\
\text { Growing } \\
(\mathbf{n}=30) \\
\text { Mean } \pm \text { SD }\end{array}$} & $\mathbf{Z}$ & $\begin{array}{c}\text { P- } \\
\text { value }\end{array}$ & \multicolumn{2}{|c|}{$\begin{array}{c}\text { Non- } \\
\text { Growing } \\
\text { Unilateral } \\
(n=14) \\
\text { Mean } \pm \\
\text { SD }\end{array}$} & \multicolumn{2}{|c|}{$\begin{array}{c}\text { Control } \\
\text { Non- } \\
\text { Growing } \\
(\mathbf{n}=\mathbf{3 0}) \\
\text { Mean } \pm \text { SD }\end{array}$} & $\mathbf{Z}$ & $\begin{array}{c}\text { P- } \\
\text { valu } \\
\text { e }\end{array}$ \\
\hline & 1.68 & 7.61 & $\begin{array}{c}30.3 \\
4\end{array}$ & 5.34 & $\begin{array}{c}4.62 \\
6\end{array}$ & $\begin{array}{c}0.001 \\
\text { H.S }\end{array}$ & $\begin{array}{c}46.0 \\
0\end{array}$ & $\begin{array}{c}7.2 \\
9\end{array}$ & $\begin{array}{c}30.3 \\
4\end{array}$ & 5.34 & $\begin{array}{c}5.09 \\
3\end{array}$ & $\begin{array}{c}0.00 \\
1 \\
\text { H.S }\end{array}$ \\
\hline B & .44 & $\begin{array}{c}10.6 \\
8\end{array}$ & 4.54 & 5.80 & $\begin{array}{l}93 \\
8\end{array}$ & $\begin{array}{c}0.001 \\
\text { H.S }\end{array}$ & $\begin{array}{c}- \\
4.86\end{array}$ & 6.9 & 4.54 & 5.80 & $\begin{array}{c}3.60 \\
6\end{array}$ & $\begin{array}{c}0.00 \\
1 \\
\text { H.S }\end{array}$ \\
\hline A & .03 & 7.72 & 4.58 & 5.72 & 3 & & $\begin{array}{c}- \\
0.24\end{array}$ & 8 & 4.58 & 5.72 & $\begin{array}{c}2.44 \\
6\end{array}$ & $\begin{array}{l}.01 \\
S\end{array}$ \\
\hline ANB & 1.57 & 5.33 & 1.40 & 3.19 & $\begin{array}{c}0.12 \\
5\end{array}$ & & 1.78 & $\begin{array}{c}3.0 \\
9\end{array}$ & 1.40 & 3.19 & $\begin{array}{c}0.55 \\
5\end{array}$ & \\
\hline SNA & .96 & 7.59 & $\begin{array}{c}82.5 \\
5\end{array}$ & 4.50 & 2 & H.S & $\begin{array}{c}76.9 \\
4\end{array}$ & $\begin{array}{c}5.4 \\
4\end{array}$ & $\begin{array}{c}82.5 \\
5\end{array}$ & 4.50 & $\begin{array}{c}2.97 \\
6\end{array}$ & $\begin{array}{l}.00 \\
3\end{array}$ \\
\hline SNB & 4.38 & 5.63 & $\begin{array}{c}81.1 \\
5\end{array}$ & 2.63 & 3.37 & $\begin{array}{c}0.001 \\
\text { H.S }\end{array}$ & $\begin{array}{c}75.1 \\
6\end{array}$ & $\begin{array}{c}5.6 \\
9\end{array}$ & $\begin{array}{c}81.1 \\
5\end{array}$ & 2.63 & $\begin{array}{c}3.40 \\
4\end{array}$ & $\begin{array}{c}0.00 \\
1 \\
H S\end{array}$ \\
\hline GO-GN & 80.25 & 4.94 & $\begin{array}{c}83.2 \\
1\end{array}$ & 3.17 & $\begin{array}{c}2.51 \\
7\end{array}$ & 0.0 & $\begin{array}{c}78.6 \\
4\end{array}$ & $\begin{array}{c}8.6 \\
5\end{array}$ & $\begin{array}{c}83.2 \\
1\end{array}$ & 3.17 & $\begin{array}{c}1.71 \\
5\end{array}$ & $\begin{array}{l}0.09 \\
\text { N.S }\end{array}$ \\
\hline BS-S-N & $\begin{array}{c}128.4 \\
9\end{array}$ & 5.73 & $\begin{array}{c}130 . \\
35\end{array}$ & 6.57 & $\begin{array}{c}1.21 \\
9\end{array}$ & $\begin{array}{l}0 . \\
\mathrm{N} .\end{array}$ & $\begin{array}{c}128 \\
19\end{array}$ & $\begin{array}{c}4.8 \\
0\end{array}$ & $\begin{array}{c}130 . \\
35\end{array}$ & 6.57 & $\begin{array}{c}1.33 \\
6\end{array}$ & $\begin{array}{c}0.2 \\
\text { N.S }\end{array}$ \\
\hline N-ANS & 59.21 & 5.16 & $\begin{array}{c}58.1 \\
5\end{array}$ & 5.79 & $\begin{array}{c}1.31 \\
3\end{array}$ & 0.2 & $\begin{array}{c}54.9 \\
2\end{array}$ & $\begin{array}{c}9.2 \\
3\end{array}$ & $\begin{array}{c}58.1 \\
5\end{array}$ & 5.79 & $\begin{array}{c}0.15 \\
1\end{array}$ & $\begin{array}{c}0.9 \\
\text { N.S }\end{array}$ \\
\hline $\begin{array}{l}\text { ANS- } \\
\text { ME }\end{array}$ & 9.96 & $\begin{array}{c}12.0 \\
0\end{array}$ & $\begin{array}{c}70.4 \\
8\end{array}$ & 7.22 & $\begin{array}{c}3.81 \\
4\end{array}$ & $\begin{array}{c}0.001 \\
\text { H.S }\end{array}$ & $\begin{array}{c}84.0 \\
8\end{array}$ & $\begin{array}{c}0.0 \\
7\end{array}$ & $\begin{array}{c}70.4 \\
8\end{array}$ & 7.22 & $\begin{array}{c}4.13 \\
5\end{array}$ & $\begin{array}{c}0.00 \\
1 \\
\text { H.S } \\
\end{array}$ \\
\hline
\end{tabular}

From the table (4) showed the following:

There was a significant difference at $\mathrm{p}$-value (0.05) between (Non-Growing Bilateral) and (Control Non-Growing) for (MP-SN, B, A, SNA, SNB, GO-GN, \& ANS-ME) where (Z) values were significant at (0.05).

There was non-significant difference at $p$-value (0.05) between (Non-Growing Bilateral) and (Control Non-Growing) for (ANB, BS-S-N \& $\mathrm{N}$-ANS) where $(\mathrm{Z})$ values were non-significant at (0.05).
There was a significant difference at p-value (0.05) between (Non-Growing Unilateral) and (Control Non-Growing) for (MP-SN, B, A, SNA, SNB \& ANS-ME) where (Z) values were significant at $(0.05)$.

There was non-significant difference at $p$-value $(0.05)$ between (Non-Growing Unilateral) and (Control Non-Growing) for (ANB, GO-GN, NANS \& BS-S-N) where (Z) values were nonsignificant at (0.05). 
Table (5): Comparison Between Growing Bilateral Group and Growing Unilateral group \& Comparison Between Non-Growing Bilateral Group and Non-Growing Unilateral group

\begin{tabular}{|c|c|c|c|c|c|c|c|c|c|c|c|c|}
\hline $\begin{array}{c}\text { Variabl } \\
\text { e }\end{array}$ & $\begin{array}{r}\text { Gro } \\
\text { Bila } \\
(\mathbf{n}= \\
\text { Mean }\end{array}$ & $\begin{array}{l}\text { ng } \\
\text { cal } \\
\text { l) } \\
\text { SD }\end{array}$ & $\begin{array}{r}\text { Gro } \\
\text { Unila } \\
(\mathbf{n}= \\
\text { Mean }\end{array}$ & & $\mathbf{Z}$ & $\begin{array}{c}\text { P- } \\
\text { valu } \\
\text { e }\end{array}$ & $\begin{array}{r}\text { No } \\
\text { Gro } \\
\text { Bilat } \\
(\mathbf{n}= \\
\text { Mean }\end{array}$ & $\begin{array}{l}- \\
\text { ing } \\
\text { ral } \\
0 \text { ) } \\
\pm \text { SD }\end{array}$ & $\begin{array}{r}\text { No } \\
\text { Grov } \\
\text { Unila } \\
\text { (n= } \\
\text { Mean }\end{array}$ & $\begin{array}{l} \\
\text { ng } \\
\text { aral } \\
\text { 4) } \\
\text { SD }\end{array}$ & $\mathbf{Z}$ & $\begin{array}{c}\text { P- } \\
\text { valu } \\
\text { e }\end{array}$ \\
\hline MP-SN & 41.14 & 8.63 & 47.24 & 8.28 & $\begin{array}{c}1.55 \\
6\end{array}$ & $\begin{array}{c}0.1 \\
\text { N.S }\end{array}$ & 51.68 & 7.61 & 46.00 & $\begin{array}{c}7.2 \\
9\end{array}$ & $\begin{array}{c}1.52 \\
2\end{array}$ & $\begin{array}{c}0.1 \\
\text { N.S }\end{array}$ \\
\hline B & -2.60 & $\begin{array}{c}10.5 \\
4\end{array}$ & -5.80 & 7.55 & $\begin{array}{c}0.77 \\
0\end{array}$ & $\begin{array}{c}0.4 \\
\text { N.S }\end{array}$ & $\begin{array}{c}- \\
10.44\end{array}$ & $\begin{array}{c}10.6 \\
8\end{array}$ & -4.86 & $\begin{array}{c}6.9 \\
6\end{array}$ & $\begin{array}{c}0.93 \\
7\end{array}$ & $\begin{array}{l}0.4 \\
\text { N.S }\end{array}$ \\
\hline A & 5.05 & 7.62 & 0.43 & 5.68 & $\begin{array}{c}1.86 \\
1\end{array}$ & $\begin{array}{l}0.06 \\
\text { N.S }\end{array}$ & -5.03 & 7.72 & -0.24 & $\begin{array}{c}5.5 \\
8\end{array}$ & $\begin{array}{c}1.58 \\
2\end{array}$ & $\begin{array}{c}0.1 \\
\text { N.S }\end{array}$ \\
\hline ANB & 8.12 & 6.03 & 3.17 & 4.02 & $\begin{array}{c}2.51 \\
9\end{array}$ & $\begin{array}{c}0.01 \\
S\end{array}$ & 1.57 & 5.33 & 1.78 & $\begin{array}{c}3.0 \\
9\end{array}$ & $\begin{array}{c}0.35 \\
2\end{array}$ & $\begin{array}{c}0.7 \\
\text { N.S }\end{array}$ \\
\hline SNA & 82.72 & 7.14 & 76.43 & 5.22 & $\begin{array}{c}2.69 \\
6\end{array}$ & $\begin{array}{c}0.00 \\
7 \\
\text { H.S }\end{array}$ & 75.96 & 7.59 & 76.94 & $\begin{array}{c}5.4 \\
4\end{array}$ & $\begin{array}{c}0.99 \\
5\end{array}$ & $\begin{array}{l}0.3 \\
\text { N.S }\end{array}$ \\
\hline SNB & 74.60 & 5.39 & 73.25 & 4.76 & $\begin{array}{c}0.81 \\
9\end{array}$ & $\begin{array}{c}0.4 \\
\text { N.S }\end{array}$ & 74.38 & 5.63 & 75.16 & $\begin{array}{c}5.6 \\
9\end{array}$ & $\begin{array}{c}0.58 \\
6\end{array}$ & $\begin{array}{c}0.4 \\
\text { N.S }\end{array}$ \\
\hline GO-GN & 70.47 & 8.48 & 75.43 & 7.66 & $\begin{array}{c}1.97 \\
4\end{array}$ & $\begin{array}{c}0.05 \\
S\end{array}$ & 80.25 & 4.94 & 78.64 & $\begin{array}{c}8.6 \\
5\end{array}$ & $\begin{array}{c}0.08 \\
8\end{array}$ & $\begin{array}{c}0.9 \\
\text { N.S }\end{array}$ \\
\hline BS-S-N & $\begin{array}{c}128.9 \\
3\end{array}$ & 7.16 & $\begin{array}{c}128.8 \\
5\end{array}$ & 8.73 & $\begin{array}{c}0.44 \\
9\end{array}$ & $\begin{array}{c}0.7 \\
\text { N.S }\end{array}$ & $\begin{array}{c}128.4 \\
9\end{array}$ & 5.73 & $\begin{array}{c}128.1 \\
9\end{array}$ & $\begin{array}{c}4.8 \\
0\end{array}$ & $\begin{array}{c}0.76 \\
1\end{array}$ & $\begin{array}{c}0.4 \\
\text { N.S }\end{array}$ \\
\hline N-ANS & 52.46 & 7.46 & 55.81 & 7.54 & $\begin{array}{c}1.60 \\
6\end{array}$ & $\begin{array}{c}0.1 \\
\text { N.S }\end{array}$ & 59.21 & $\begin{array}{c}5.16 \\
2\end{array}$ & 54.92 & $\begin{array}{c}9.2 \\
3\end{array}$ & $\begin{array}{c}1.61 \\
1\end{array}$ & $\begin{array}{c}0.1 \\
\text { N.S }\end{array}$ \\
\hline $\begin{array}{c}\text { ANS- } \\
\text { ME }\end{array}$ & 69.45 & $\begin{array}{c}12.9 \\
7\end{array}$ & 74.57 & $\begin{array}{c}10.7 \\
0\end{array}$ & $\begin{array}{c}1.10 \\
7\end{array}$ & $\begin{array}{c}0.3 \\
\text { N.S }\end{array}$ & 89.96 & $\begin{array}{c}12.0 \\
0\end{array}$ & 84.08 & $\begin{array}{c}8.8 \\
7\end{array}$ & $\begin{array}{c}1.87 \\
4\end{array}$ & $\begin{array}{l}0.06 \\
\text { N.S }\end{array}$ \\
\hline
\end{tabular}

HS significant at (0.01)

From the table (5) showed the following:

There was a significant difference at $p$-value (0.05) between (Growing Bilateral) and (Growing Unilateral) for (ANB, SNA \& GOGN) where $(Z)$ values were significant at (0.05).

There was non-significant difference at $p$-value (0.05) between (Growing Bilateral) and (Growing Unilateral) for (MP-SN, B, A, SNB, BS-S-N, N-ANS \& ANS-ME) where (Z) values were non-significant at (0.05).

There was non-significant difference at $p$-value (0.05) between (Non-Growing Bilateral) and (Non-Growing Unilateral) for (MP-SN, B, A, ANB, SNA, SNB, GO-GN, BS-S-N, N-ANS \& ANS-ME) where ( $Z$ ) values were nonsignificant at (0.05).

\section{Discussion}

In our study, we chosen non-operated patients with no surgical intervention to concentrate on the natural morphology of the craniofacial structure and to exclude any other external factor such as surgery. Many previous studies conducted on operated adults ${ }^{[32,38]}$, operated young age ${ }^{[39]}$ or non-operated young age ${ }^{[9,29]}$ few studies operated on wide age group [40] .The current study is conducted on both growing young patients and non-growing adults both unilateral and bilateral cleft lip and palate.

The results of this study demonstrated that craniofacial morphology changes in UCLP growing patients were significantly different from normal growing patients in; (MP-SN), (SNA), (SNB), (GO-GN), (N-ANS) and (ANS$\mathrm{ME})$. On the other hand, we noticed normal 
(Bs-S-N) similar to control group. This is in agreement with previous observations ${ }^{[40,41]}$

According to the current study, it can be concluded that growth and development of upper face was reduced in UCLP growing patients. The reason behind this finding was discussed by Nielsen et al ${ }^{[42]}$ who attributed the abnormal upper facial morphology in UCLP to that; cleft individuals had high incidence of deviation of Sella turcica morphology, which is the key point for the neural crest cells to migrate to the frontonasal and maxillary growth field, this abnormality in Sella turcica growth can cause abnormal maxillary morphology. Another explanation for abnormal facial morphology in UCLP patients may be the splitting of the palate from nasal septum ${ }^{[43]}$.

Moreover, the results of the current study showed that the cephalometric measurements changes in BCLP growing patients were significantly different from normal individual in; (MP-SN), (A, B points), (ANB), (SNB) and (ANS-Me).

Shreshta et al 2018, who examine the relationship between mandibular volume and craniofacial morphology in young patient with cleft lip and palate using CBCT. They attain similar results regarding the correlation position of maxilla to mandible (ANB), MP$\mathrm{SN}$ and the point $\mathrm{B}$ when comparing the BCLP growing and control growing patients. This may be explained by the abnormal growth pattern of maxilla in patients with CLP, that affects the shape of maxilla greatly. Moreover, most patients with CLP exhibit mouth breathing due to airway abnormalities thus mouth breathing increase the lower face height (ANS-Me) resulting in subsequent posterior rotation of the mandible affecting the position of the point $\mathrm{B}$ of the mandible and the ANB angle ${ }^{[44,45]}$.

The MP-SN measurement was larger in both the BCLP growing group and UCLP growing group than in control groups. Similar results were obtained by Hermann et al ${ }^{[44]}$ who believed that primary factors, such as cleft malformation, or secondary factors, such as development and functional adaptations, caused this. The increased inclination of the mandible to the anterior cranial base is a type of mandibular vertical compensatory growth in response to horizontal and vertical changes seen in the midfacial region in patients with mandibular vertical compensation.

Our results showed that significant differences between BCLP growing and UCLP growing groups were only in (ANB), (SNA) and (GOGN) which showing shorter mandibular length in BCLP growing group than UCLP growing group.

The present study showed larger SNA in BCLP patients than in UCLP and control patients, in additions ANB was larger in BCLP group than UCLP and control groups, similar results attained by shreshta et al 2018 .

On the contrary to our results, Eriksen et al observed that patients with more extensive clefts had a significantly shorter mandibular length ${ }^{[46]}$

However, in 1982 Long and col. suggested that patients with cleft lip and palate and severe maxillary retrusion may be considered as a result of a more severe fissure and in order to compensate for the maxillary deficiency, the mandibular size increases. ${ }^{[4]}$ In our study, we noticed increase in GO-GN length of the mandible in both BCLP growing patients and in UCLP growing patients more than control group and this results in accordance with Yudovich Burak et al ${ }^{[38]}$ who found $82.76 \%$ of their patients had an increase of the mandibular body length.

The results of this study comparing the nongrowing UCLP group and BCLP group to control non-growing group demonstrated that there were significant differences in; (MP-SN), B point, A point, (SNA), (SNB) and (ANS$\mathrm{Me}$ ). Only GO-GN mandibular length was significant in BCLP non-growing patients when compared to control non-growing group of patients.

These results showed some differences as well as some similarities with the results obtained 
by Yi. Xu et al 2014, as they found normal values of SNA in adulthood, in addition, they showed normal mandibular length (GO-GN) in UCLP adult patients.

The growth and development of the mandible in UCLP non-growing patients seems to be postponed and so patients will eventually show normal mandibular morphology in adulthood, this conclusion also believed by Smahel $1984^{[48]}$ as he said that changes of mandibular shape represented adaptation secondary to cleft palate.

Our study showed significant difference in mandibular length in non- growing BCLP group in relation to normal patients which may be due to failure of the mandible to adapt and to develop normally due to severity of the cleft. The length of upper facial height seems to catch up later in growth process as its values for non-growing group where normal in relation to normal control groups

The cranial base divergence (BS-S-N) for all test patients' group, growing and non-growing BCLP and UCLP groups demonstrated a normal value similar to the control groups and this in agree with previous studies [41, 49, 50]. also, these results were similar to the results of $\mathrm{Y}$. Xu et $\mathrm{al}^{[40]}$ who found normal cranial base angle in UCLP in Childhood and adult patients but with shortening of the pre- and post-sellar length and they concluded that growth and development of the cranial base in UICP patients were proportionally reduced.

The relation between changes in cranial base morphology and cleft palate can be explained in both anatomical and functional terms, Anatomically, the cranial base can be seen as a boundary structure between the neurocranium and the facial skeleton, therefore, the development and growth of the cranial base can interfere with both neurocranial and facial skeletal development ${ }^{[50]}$.

Functionally, factors such as prolonged head posture due to reduced airway size can also influence the postnatal growth pattern of the cranial base in subjects with ICP [41]

In the current study, we use $3 \mathrm{D}$ cephalometric analysis obtained from CBCT, many authors found that reproducibility of the cephalometric measurements obtained from CBCT was higher than cephalometric measurements of conventional cephalometric radiograph ${ }^{[51-55]}$.

\section{Conclusion}

The study operated on a wide age groups of non-operated UCLP and BCLP patients to concentrate on the morphology of the craniofacial structure and to exclude any other external factor such as surgery. Our study shows that there was significant increase in mandibular length in both growing and nongrowing BCLP than of UCL, in growing UCLP there was reduced upper face height and in growing BCLP there was increased lower face height. This needs to be considered during orthodontic treatment planning and surgical management for the cleft defect.

\section{References}

1. Vellappally, S., et al., The prevalence of malocclusion and its association with dental caries among 12-18-year-old disabled adolescents. BMC Oral Health, 2014. 14: p. 123.

2. Shaye, D., C.C. Liu, and T.T. Tollefson, Cleft Lip and Palate: An EvidenceBased Review. Facial Plast Surg Clin North Am, 2015. 23(3): p. 357-72.

3. Gupta, A., et al., Assessing Angle's malocclusion among cleft lip and/or palate patients in Jammu. J Int Soc Prev Community Dent, 2016. 6(Suppl 1): p. S23-7.

4. Kianifar, H., et al., Cleft lip and Palate: A 30-year Epidemiologic Study in North-East of Iran. Iran J Otorhinolaryngol, 2015. 27(78): p. 35-41.

5. Abulezz, T.A., A.K. Elsherbiny, and A.S. Mazeed, Management of cleft lip and palate in Egypt: A National survey. Indian J Plast Surg, 2018. 51(3): p. 290-295.

6. Parameters for evaluation and treatment of patients with cleft lip/palate or other craniofacial anomalies. American Cleft PalateCraniofacial Association. March, 1993. Cleft Palate Craniofac J, 1993. 30 Suppl: p. S1-16.

7. M, B. and D. AS, Epidemiologyclinical profile of cleft lip and palate among children in India and its surgical consideration. CJS, 2013. 2: p. 45-51. 
8. Williams, A.C., et al., The surgical care of cleft lip and palate patients in England and Wales. Br J Plast Surg, 1996. 49(3): p. 150-5.

9. Antonarakis, G.S., B.D. Tompson, and D.M. Fisher, Preoperative Cleft Lip Measurements and Maxillary Growth in Patients With Unilateral Cleft Lip and Palate. Cleft Palate Craniofac J, 2016. 53(6): p. e198e207.

10. Chiu, Y.T. and Y.F. Liao, Is cleft severity related to maxillary growth in patients with unilateral cleft lip and palate? Cleft Palate Craniofac J, 2012. 49(5): p. 535-40.

11. Chiu, Y.T., Y.F. Liao, and P.K. Chen, Initial cleft severity and maxillary growth in patients with complete unilateral cleft lip and palate. Am J Orthod Dentofacial Orthop, 2011. 140(2): p. 189-95.

12. Hsieh, Y.J., Y.F. Liao, and A. Shetty, Predictors of poor dental arch relationship in young children with unilateral cleft lip and palate. Clin Oral Investig, 2012. 16(4): p. 1261-6.

13. Tomita, Y., et al., Severity of alveolar cleft before palatoplasty affects vertical maxillofacial growth in 6-year-old patients with complete unilateral cleft lip and palate. Am J Orthod Dentofacial Orthop, 2012. 141(4 Suppl): p. S102-9.

14. Liao, Y.F. and M. Mars, Long-term effects of palate repair on craniofacial morphology in patients with unilateral cleft lip and palate. Cleft Palate Craniofac J, 2005. 42(6): p. 594-600.

15. Liao, Y.F., et al., Cleft size at the time of palate repair in complete unilateral cleft lip and palate as an indicator of maxillary growth. Int J Oral Maxillofac Surg, 2010. 39(10): p. 956-61.

16. Meazzini, M.C., et al., Maxillary growth impairment in cleft lip and palate patients: a simplified approach in the search for a cause. J Craniofac Surg, 2008. 19(5): p. 1302-7.

17. Meazzini, M.C., et al., Factors that affect variability in impairment of maxillary growth in patients with cleft lip and palate treated using the same surgical protocol. $\mathrm{J}$ Plast Surg Hand Surg, 2011. 45(4-5): p. 18893.
18. Wiggman, K., et al., The influence of the initial width of the cleft in patients with unilateral cleft lip and palate related to final treatment outcome in the maxilla at 17 years of age. Eur J Orthod, 2013. 35(3): p. 335-40.

19. Reiser, E., et al., Association between cleft size and crossbite in children with cleft palate and unilateral cleft lip and palate. Cleft Palate Craniofac J, 2010. 47(2): p. 175-81.

20. Baumrind, S. and R.C. Frantz, The reliability of head film measurements. 2. Conventional angular and linear measures. Am J Orthod, 1971. 60(5): p. 505-17.

21. Baumrind, S. and R.C. Frantz, The reliability of head film measurements. 1 . Landmark identification. Am J Orthod, 1971. 60(2): p. 111-27.

22. Pittayapat, P., et al., Three-dimensional cephalometric analysis in orthodontics: a systematic review. Orthod Craniofac Res, 2014. 17(2): p. 69-91.

23. Maspero, C., et al., Assessing mandibular body changes in growing subjects: a comparison of CBCT and reconstructed lateral cephalogram measurements. Sci Rep, 2020. 10(1): p. 11722.

24. Afghari P, G.R., Sohilipour S. , Anatomical assessment of foramen tympanicum using cone beam computed tomography images. Dent Hypotheses, 2016. 7: p. 107.

25. Suomalainen A, K.T., Kaser Y, Peltola $\mathrm{J}$, Kortesniemi $\mathrm{M}$, Dosimetry and image quality of four dental cone beam computed tomography scanners compared with multislice computed tomography scanners. Dentomaxillofac Radiol 2009. 38: p. 367-78.

26. Hashimoto K, K.S., Araki M, Iwai K, Sawada K, Akiyama Y. , Comparison of image performance between cone-beam computed tomography for dental use and four-row multidetector helical CT. J Oral Sci 2006. 48: p. 27-34.

27. Scarfe WC, F.A., Sukovic P. , Clinical applications of cone-beam computed tomography in dental practice. J Can Dent Assoc, 2006. 72: p. 75-80.

28. Cattaneo, P.M. and B. Melsen, The use of cone-beam computed tomography in an orthodontic department in between research 
and daily clinic. World J Orthod, 2008. 9(3): p. 269-82.

29. Shrestha, A., et al., Three-dimensional evaluation of mandibular volume in patients with cleft lip and palate during the deciduous dentition period. Angle Orthod, 2020. 90(1): p. 85-91.

30. Machado, G.L., CBCT imaging - $A$ boon to orthodontics. Saudi Dent J, 2015. 27(1): p. 12-21.

31. Kapila, S.D. and J.M. Nervina, $C B C T$ in orthodontics: assessment of treatment outcomes and indications for its use. Dentomaxillofac Radiol, 2015. 44(1): p. 20140282.

32. Buyuk, S.K., et al., Evaluation of the Transverse Craniofacial Morphology of Adolescents With Repaired Unilateral Cleft Lip and Palate Using Cone-Beam Computed Tomography. J Craniofac Surg, 2016. 27(7): p. 1870-1874.

33. Kumar, V., et al., In vivo comparison of conventional and cone beam CT synthesized cephalograms. Angle Orthod, 2008. 78(5): p. 873-9.

34. Zamora, N., et al., A study on the reproducibility of cephalometric landmarks when undertaking a three-dimensional (3D) cephalometric analysis. Med Oral Patol Oral Cir Bucal, 2012. 17(4): p. e678-88.

35. Lagravère, M.O., et al., Reliability of traditional cephalometric landmarks as seen in three-dimensional analysis in maxillary expansion treatments. Angle Orthod, 2009. 79(6): p. 1047-56.

36. Farronato, M., et al., 3D cephalometry on reduced FOV CBCT: skeletal class assessment through $A F-B F$ on Frankfurt plane-validity and reliability through comparison with $2 D$ measurements. European Radiology, 2020. 30(11): p. 6295-6302.

37. Power, G., et al., Dolphin Imaging Software: An analysis of the accuracy of cephalometric digitization and orthognathic prediction. International journal of oral and maxillofacial surgery, 2005. 34: p. 619-26.

38. Yudovich Burak, M., A. Ponglertnapakorn, and E.G. García Calderón, Analysis of the cephalometric skeletal and dental characteristics of adult patients with cleft lip and palate who received orthopedic, orthodontic and/or surgical treatment during their childhood and adolescence. Revista Mexicana de Ortodoncia, 2015. 3(1): p. e22e32.

39. Kochhar, A.S., et al., Frontal and Axial Evaluation of Craniofacial Morphology in Repaired Unilateral Cleft Lip and Palate Patients Utilizing Cone Beam Computed Tomography; An Observational Study. Int J Environ Res Public Health, 2020. 17(21).

40. $\mathrm{Xu}, \mathrm{Y}$., et al., Cephalometric analysis of craniofacial morphology and growth in unrepaired isolated cleft palate patients. J Craniomaxillofac Surg, 2014. 42(8): p. 185360.

41. Hermann, N.V., et al., Early craniofacial morphology and growth in children with unoperated isolated cleft palate. Cleft Palate Craniofac J, 2002. 39(6): p. 60422.

42. Nielsen, B.W., K. Mølsted, and I. Kjaer, Maxillary and sella turcica morphology in newborns with cleft lip and palate. Cleft Palate Craniofac J, 2005. 42(6): p. 610-7.

43. Smahel, Z. and M. Brejcha, Differences in craniofacial morphology between complete and incomplete unilateral cleft lip and palate in adults. Cleft Palate J, 1983. 20(2): p. 113-27.

44. Hermann, N.V., et al., Early Craniofacial Morphology and Growth in Children with Bilateral Complete Cleft Lip and Palate. The Cleft Palate-Craniofacial Journal, 2004. 41(4): p. 424-438.

45. van den Dungen, G.M., et al., Craniofacial morphology of Dutch patients with bilateral cleft lip and palate and noncleft controls at the age of 15 years. Cleft Palate Craniofac J, 2008. 45(6): p. 661-6.

46. Eriksen, J., et al., Early Postnatal Development of the Mandible in Children with Isolated Cleft Palate and Children with Nonsyndromic Robin Sequence. The Cleft Palate-Craniofacial Journal, 2006. 43(2): p. 160-167.

47. Long, R.E., R.B. Jain, and W.M. Krogman, Possible sex-discriminant variables in craniofacial growth in clefting. American Journal of Orthodontics, 1982. 82(5): p. 392402. 
48. Smahel, Z., Variations in craniofacial morphology with severity of isolated cleft palate. Cleft Palate J, 1984. 21(3): p. 140-58.

49. Dahl, E., et al., Comparison of craniofacial morphology in infants with incomplete cleft lip and infants with isolated cleft palate. Cleft Palate J, 1982. 19(4): p. 25866.

50. Mølsted, K., I. Kjaer, and E. Dahl, Cranial base in newborns with complete cleft lip and palate: radiographic study. Cleft Palate Craniofac J, 1995. 32(3): p. 199-205.

51. van Vlijmen, O.J., et al., A comparison between $2 D$ and $3 D$ cephalometry on CBCT scans of human skulls. Int J Oral Maxillofac Surg, 2010. 39(2): p. 156-60.

52. Lee, M., G. Kanavakis, and R.M. Miner, Newly defined landmarks for a threedimensionally based cephalometric analysis: a retrospective cone-beam computed tomography scan review. Angle Orthod, 2015. 85(1): p. 310.

53. van Vlijmen, O.J., et al., Comparison of cephalometric radiographs obtained from cone-beam computed tomography scans and conventional radiographs. J Oral Maxillofac Surg, 2009. 67(1): p. 92-7.

54. Periago, D.R., et al., Linear accuracy and reliability of cone beam $C T$ derived 3dimensional images constructed using an orthodontic volumetric rendering program. Angle Orthod, 2008. 78(3): p. 387-95.

55. Medelnik, J., et al., Accuracy of anatomical landmark identification using different CBCT- and MSCT-based 3D images: an in vitro study. J Orofac Orthop, 2011. 72(4): p. 261-78. 